\title{
Studies on Agricultural Development Based on Optimized Combination Forecasting and Markov Model
}

\author{
Zhang Yuting ${ }^{1}$ \\ 1. Department of Mathmaticis, Jinan University \\ Guangzhou, China \\ zytjnu@qq.com
}

\begin{abstract}
Taking Shunde District, China as an example, we analyze the development of agriculture using the optimal combination of the grey system forecasting model, linear regression model, and polynomial regression model. The results show that the optimized combination forecasting model has feasibility and practicability, and has practical significance for the research on agricultural development. And then, we use the Markov model to further study the structure of the production value of agriculture. And the results show that the model effectively predicts the development of the structure of the production value of agriculture. Based upon the above studies and our records of field interviews in Beijiao Town, Shunde District, we explore the bottlenecks in the process of agricultural development in Shunde District, and propose scientific and practical suggestions.
\end{abstract}

Keywords-grey system forecasting; regression forecasting; optimized combination forecasting; Markov model

\section{INTRODUCTION}

As the country's basis, agriculture is the foundation of the national economy and is closely related to people's life. With the development of science and technology, the development of agricultural economy is also great rapid. Taking Shunde District of Foshan, Guangdong, China as an example, according to the data in the Shunde Statistical Yearbook published by the Development Planning Statistics Bureau of Shunde District of Foshan, the total agricultural output value in Shunde in 2007 was 6.141 billion yuan, and the total agricultural output value in 2010 was 7.042 billion yuan, which rose by $14.67 \%$. By 2015 , the total agricultural output value reached 8.602 billion yuan, which rose by $40.07 \%$. The growth rate of the total agricultural output value of country must be even more rapid. The development of agriculture not only relates to people's living standards, but also guarantees the country's stability. Therefore, studying the future development trend of agricultural is such a practical and significant matter.

In this paper, we comprehensively considers the existing studies to study on agricultural development. First we adopt a combination of grey system forecasting, liner regression and polynomial regression as optimized combination forecasting to predict and analyze the development of agricultural output value. Second, we use Markov prediction model to study the development of agricultural industrial structure. In addition, in order to find out the problems that may be encountered in the development of agriculture and correspond to the bottlenecks in the prediction, we select Beijiao Town of Shunde as a

This work was supported by the Fundamental Research Funds for the Central Universities (Grant No. 21617417). research site, interview the local people and do some field surveys. Combining the problems found in research and analysis, we give some corresponding suggestions.

\section{ForecAst of Agricultural OUtPut VAlue}

In order to predict the future development of agriculture in Shunde District, we combine the following three models: grey system (GM) forecasting model based on grey system theory, linear regression, and polynomial regression model. And the data is from the Statistical Yearbook published by the Development Planning Statistics Bureau of Shunde District of Foshan.

\section{A. GM Model}

\section{1) Introduction to Model}

The grey system theory, which was proposed by Prof. Deng Julong in 1982, is a mathematical method to solve incomplete information systems. This method is mainly used to study the lean information modeling, and provides a new way to solve system problems with very little information. Therefore, GM model has the advantage when we only have few samples.

In agricultural development, the agricultural output value system contains both known information and unknown information. Therefore, this system can be regarded as a grey system between black system and white system. In this regard, we use GM model to predict the development of agricultural output value.

\section{2) Introduction to GM model}

When establishing GM forecasting model, it's necessary to check whether the data meets the modeling requirements. For the original data $\mathrm{X}^{(0)}=\left\{\mathrm{X}^{(0)}(1), \ldots, \mathrm{X}^{(0)}(\mathrm{n})\right\}$, the series ratio is defined by $\sigma(\mathrm{k})=\mathrm{x}^{0}(\mathrm{k}-1) / \mathrm{x}^{0}(\mathrm{k})$. If the series ratio $\sigma(k) \in\left(e^{-2 /(n+1)}, e^{2 /(n+1)}\right)$, then the data can be used for modeling. For data meets the modeling requirements, we can construct GM model to further analysis.

Accumulate $X^{(0)}$ once to get $X^{(1)}=\left\{x^{(1)}(1), \ldots, x^{(1)}(n)\right\}$, where

$$
x^{(1)}(k)=\sum_{i=0}^{k} x^{(0)}(i)
$$

Then, the differential formula of $G M(1.1)$ expression of $x^{(0)}(k)$ is

$$
\frac{d x^{(1)}}{d t}+a x^{(1)}=u,
$$

where $\mathrm{a}, \mathrm{u}$ are parameters to be determined. 
Let $Y=\left[\begin{array}{c}x^{(0)}(2) \\ x^{(0)}(3) \\ \vdots \\ x^{(0)}(n)\end{array}\right], \mathrm{B}=\left[\begin{array}{cc}-0.5\left(\mathrm{x}^{(1)}(1)+\mathrm{x}^{(1)}(2)\right) & 1 \\ -0.5\left(\mathrm{x}^{(1)}(2)+\mathrm{x}^{(1)}(3)\right) & 1 \\ \vdots & \vdots \\ -0.5\left(\mathrm{x}^{(1)}(\mathrm{n}-1)+\mathrm{x}^{(1)}(\mathrm{n})\right) & 1\end{array}\right]$,

$\Phi=\left[\begin{array}{ll}a & u\end{array}\right]^{T}$. Then, we have $Y=B \Phi$. By the least square method, we obtain the estimation of $\Phi$

$$
\widehat{\Phi}=[\hat{a}, \hat{u}]^{T}=\left(B^{T} B\right)^{-1} B^{T} Y
$$

The discrete solution to the differential equation (3) is

$$
\hat{x}^{(1)}(k+1)=\left[x^{(1)}(1)-\frac{\hat{u}}{\hat{a}}\right] e^{-\hat{a} k}+\frac{\widehat{u}}{\hat{a}}
$$

From formula (1) and (4), revert to the original data

$$
\hat{x}^{(0)}(k+1)=\left(1-e^{\hat{a}}\right)\left[x^{(1)}(1)-\frac{\widehat{u}}{\hat{a}}\right] e^{-\hat{a} k}
$$

\section{3) Error Estimation of GM Model}

Obtain $\hat{X}^{(1)}$ by formula (4), and obtain $\hat{X}^{(0)}$ by formula (5), calculate the residual as $E=[e(2), e(3), \ldots, e(n)]=$ $X^{(0)}-\hat{X}^{(0)}$, where $e(k)=x^{(0)}(k)-\hat{x}^{(0)}(k), k=1,2, \ldots, n$.

Let $S_{1}^{2}$ and $S_{2}^{2}$ be the variances of $X^{(0)}$ and $E$ respectively, that is,

$$
\begin{gathered}
S_{1}^{2}=\frac{1}{n} \sum_{k=1}^{n}\left[x^{(0)}(k)-\bar{x}\right]^{2}, \text { where } \bar{x}=\frac{1}{n} \sum_{k=1}^{n} x^{(0)}(k) \\
S_{2}^{2}=\frac{1}{n} \sum_{k=2}^{n}[e(k)-\bar{e}]^{2}, \text { where } \bar{e}=\frac{1}{n} \sum_{k=2}^{n} e(k)
\end{gathered}
$$

The posterior difference ratio and the error probability are

$$
C=S_{2} / S_{1}, \quad p=P\left\{|e(k)-\bar{e}|<0.6746 S_{1}\right\}
$$

The following table is a reference table for GM model accuracy inspection level:

TABLE I. ACCURACY INSPECTION LEVEL REFERENCE TABLE

\begin{tabular}{ccc}
\hline \hline Model accuracy level & $\boldsymbol{C}$ & $\boldsymbol{p}$ \\
\hline Level 1 (good) & $C \leq 0.35$ & $0.95 \leq p$ \\
Level 2 (qualified) & $0.35<C \leq 0.5$ & $0.80 \leq p<0.95$ \\
Level 3 (reluctant) & $0.5<C \leq 0.65$ & $0.70 \leq p<0.80$ \\
Level 4(unqualified) & $0.65<C$ & $p<0.70$ \\
\hline$G M$ Model Test & &
\end{tabular}

According to the data published by the Statistical Yearbook, we use GM model to predict the development of agricultural output value in Shunde District:

$$
\hat{x}^{(1)}(k+1)=-1625.43 e^{0.0383 k}-1686.8370
$$

And the sequence $\hat{x}^{(0)}$ can be obtained by

$$
\hat{x}^{(0)}(k+1)=\hat{x}^{(1)}(k+1)-\hat{x}^{(1)}(k)
$$

According to this model, we predict the agricultural output value of Shunde District from 2007 to 2015.
TABLE II.

GM MODEL RESULTS (100 MILLION YUAN)

\begin{tabular}{cccc}
\hline Year & $\begin{array}{c}\text { Actual agricultural } \\
\text { output value }\end{array}$ & $\begin{array}{c}\text { Predicted agricultural } \\
\text { output value }\end{array}$ & Relative error \\
\hline 2007 & 61.41 & 68.24 & 0.0099 \\
2008 & 68.92 & 70.90 & -0.0374 \\
2009 & 68.35 & 73.67 & -0.0462 \\
2010 & 70.42 & 76.55 & 0.0220 \\
2011 & 78.27 & 79.53 & 0.0429 \\
2012 & 83.1 & 82.64 & 0.0477 \\
2013 & 86.78 & 85.86 & -0.0132 \\
2014 & 84.75 & 89.22 & -0.0372 \\
2015 & 86.02 & & \\
\hline
\end{tabular}

The error estimate for the model in this case is

$S 1=77.7324, S 2=7.7301, C=0.099445, p=1$.

As the result shows, this model is suitable for agricultural output value forecasting.

\section{B. Linear Regression Model}

\section{1) Introduction to Linear Regression}

As a commonly used method in mathematical statistics, linear regression analysis is one of the simplest and most commonly used methods to predict the development of systems, and is also the most effective one. For the agricultural development system, agricultural output value is obviously inextricably linked with time. Therefore, linear regression is usually a very common method for predicting the agricultural output value.

Suppose there are $n$ sets of data $\left(x_{1}, y_{1}\right), \ldots,\left(x_{n}, y_{n}\right)$, and the linear regression formula is $\hat{y}_{i}=a+b x_{i}$. Thereby, the prediction error is

$$
e_{i}=y_{i}-\hat{y}_{i}
$$

In the linear regression model, the best $(a, b)$ should minimize the sum of squared error

$$
Q=\sum_{i=1}^{n} e_{i}^{2}=\sum_{i=1}^{n}\left(y_{i}-a-b x_{i}\right)^{2} \text {. }
$$

Hence we have

$$
\begin{gathered}
a=\frac{1}{n} \sum_{i=1}^{n} y_{i}-\frac{b}{n} \sum_{i=1}^{n} x_{i}=\bar{y}-b \bar{x} \\
b=\frac{\sum x_{i} y_{i}-\bar{y} \sum x_{i}}{\sum x_{i}{ }^{2}-\bar{x} \sum x_{i}}=\frac{\sum x_{i} y_{i}-n \bar{x} \bar{y}}{\sum x_{i}{ }^{2}-n \bar{x}^{2}}
\end{gathered}
$$

From formula (6), we obtain the forecasting model

$$
\hat{y}_{i}=a+b x_{i}
$$

\section{2) Linear Regression Model Test}

According to the data published by the Statistical Yearbook, we use linear regression model to predict the development of agricultural output value in Shunde District, and obtain that

$$
a=60.1575, \quad b=3.2578 \text {. }
$$

Therefore, in this case, formula (7) is

$$
\hat{y}_{i}=60.1575+3.2578 x_{i}
$$

According to this model, we predict the agricultural output value of Shunde District from 2007 to 2015 in the following:

TABLE III. LINEAR REGRESSION RESULTS (100 MILLION YUAN) 


\begin{tabular}{cccc}
\hline \hline Year & $\begin{array}{c}\text { Actual agricultural } \\
\text { output value }\end{array}$ & $\begin{array}{c}\text { Predicted agricultural } \\
\text { output value }\end{array}$ & Relative error \\
\hline 2007 & 61.41 & & \\
2008 & 68.92 & 67.68 & 0.0181 \\
2009 & 68.35 & 70.72 & -0.0347 \\
2010 & 70.42 & 73.76 & -0.0475 \\
2011 & 78.27 & 76.80 & 0.0187 \\
2012 & 83.1 & 79.85 & 0.0391 \\
2013 & 86.78 & 82.89 & 0.0448 \\
2014 & 84.75 & 85.93 & -0.0140 \\
2015 & 86.02 & 88.98 & -0.0344 \\
\hline
\end{tabular}

It can be seen that the error of this model is acceptable, and the result is closer to the real data.

\section{Polynomial Regression Model}

\section{1) Introduction to Polynomial Regression}

Linear regression model is based on the basic assumption that there is a linear relationship between variables. In fact, the relationship between variables in economic development is mostly non-linear. For agricultural development forecasting, nonlinear regression model can be used to balance the information loss that linear regression may bring.

Suppose there are $n$ sets of data $\left(x_{1}, y_{1}\right), \ldots,\left(x_{n}, y_{n}\right)$, and the polynomial regression formula is

$$
\widehat{y}_{l}=\beta_{0}+\beta_{1} x_{i}+\beta_{2} x_{i}^{2}+\cdots+\beta_{n} x_{i}^{n}
$$

\section{2) Polynomial Regression Model Test}

According to the data published by the Statistical Yearbook, we use the polynomial regression model to predict the development of agricultural output value in Shunde, since other paprameter is too small to have little impact on the predictions, we obtain that

$$
\beta_{0}=55.9402, \quad \beta_{1}=5.5582, \quad \beta_{2}=0.2300 .
$$

Therefore, in this case, formula (8) is

$$
\widehat{y}_{i}=55.9402+5.5582 x_{i}-0.2300 x_{i}^{2}
$$

According to this model, we predict the agricultural output value of Shunde District from 2007 to 2015 in the following:

TABLE IV. POLYNOMIAL REGRESSION RESUlTS (100 MiLLION YUAN)

\begin{tabular}{cccc}
\hline Year & $\begin{array}{c}\text { Actual agricultural } \\
\text { output value }\end{array}$ & $\begin{array}{c}\text { Predicted agricultural } \\
\text { output value }\end{array}$ & Relative error \\
\hline 2007 & 61.41 & 65.98 & 0.0427 \\
2008 & 68.92 & 70.48 & -0.0311 \\
2009 & 68.35 & 74.49 & -0.0578 \\
2010 & 70.42 & 78.02 & 0.0032 \\
2011 & 78.27 & 81.06 & 0.0245 \\
2012 & 83.1 & 83.62 & 0.0364 \\
2013 & 86.78 & 85.69 & -0.0111 \\
2014 & 84.75 & 87.28 & -0.0146 \\
2015 & 86.02 & & \\
\hline
\end{tabular}

It can be seen that the error of this model is acceptable, and the result is closer to the real data. Compare to the result from the linear regression model, we find that sometimes the polynomial regression is better than linear regression, while sometimes linear regression is better.

\section{Optimized Combination Forecasting Model}

\section{1) Introduction to Model}

For economic development forecasting, various methods have their own advantages and disadvantages, and each of them can achieve certain results in predicting. However, if we analyze from a single aspect, we will often ignore too many factors and might create a model that the prediction results are not accurate and reliable. As for this, J.M.Bates and C.W.J.Granger proposed the theory and method of combined forecasting in 1969. For a forecasting problem, combination forecasting is to combine several forecasting methods with weighted average method. In this way, under the combination forecasting, even if a certain model's predictive performance is poor, the forecasting system's performance still can in a relatively stable and good state due to the system's nature.

In this paper, we build an optimized combination model consist of GM forecasting model, linear regression model and polynomial regression model, to improve the practicality and reliability of the model.

\section{2) Model Basics}

Suppose that there are $n$ prediction models for a prediction problem, let $K_{i}$ be the weighting coefficient of the $i^{\text {th }}(i=1,2, \ldots, n)$ prediction model, and $\sum K_{i}=1$. Then, the combination forecasting model is

$$
f=\sum_{i=1}^{n} K_{i} f_{i t}
$$

$f$ is the predicted value of the combination forecasting model, and $f_{i}$ is the predicted value of the $i^{\text {th }}$ prediction model. Note the prediction error at $t(t=1,2, \ldots, m)$ of the $i^{\text {th }}(i=1,2, \ldots, n)$ prediction model as

$$
\begin{gathered}
e_{i t}=y_{i t}-\hat{y}_{i t} \quad i=1,2, \ldots, n, t=1,2, \ldots m \\
E_{i}=\sum_{t=1}^{m} e_{i t}{ }^{2} \quad i=1,2, \ldots, n
\end{gathered}
$$

In this paper, the method to calculate the weights in combination forecasting is as below

$$
K_{i}=\frac{1}{n-1} \times \frac{\sum_{j=1}^{n} E_{j}-E_{i}}{\sum_{j=1}^{n} E_{j}} \quad i=1,2, \ldots, n
$$

\section{3) Forecast of Future Agricultural Development}

For the three-combined forecasting model, based on the formula (10), we have

$$
K_{1}=0.3121, \quad K_{2}=0.3289, \quad K_{3}=0.3590
$$

With formula (9), predict the future agricultural output value in Shunde as the following table.

TABLE V. OPTIMIZED COMBINATION FORECASTING RESULTS

\begin{tabular}{cccc}
\hline \hline Year & $\begin{array}{c}\text { Agricultural } \\
\text { practitioners }\end{array}$ & $\begin{array}{c}\text { Agricultural output } \\
\text { value }\end{array}$ & $\begin{array}{c}\text { Average output } \\
\text { value (yuan) }\end{array}$ \\
\hline 2016 & 51377.03 & 90.92 & 121465.96 \\
2017 & 48254.36 & 93.28 & 124607.06 \\
2018 & 45119.19 & 95.50 & 127574.31 \\
2019 & 41969.54 & 97.59 & 130369.96 \\
2020 & 38803.49 & 99.55 & 132996.38 \\
\hline
\end{tabular}

From the analysis result of the three models, combination forecasting model is more accurate, feasible and practical. From the forecast results, agricultural practitioners continuously decrease while the agriculture economy continuously promote. The development of agricultural economy in Shunde still has potential for further expansion. However, the agricultural practitioners' issues will continue decrease. Meanwhile, the agricultural economy of Shunde is gradually encountering bottleneck and the development trend 
will be curbed. Therefore, it's of great necessary to find out the bottleneck of agricultural development in Shunde.

\section{Prediction OF Agricultural Industry StruCture}

In order to find the bottleneck in the agricultural development in Shunde, we studied the agricultural industrial structure by using Markov model.

\section{A. Introduction to Markov Model}

At the beginning of $20^{\text {th }}$ century, Markov, a Russian mathematician, proposed Markov prediction method. A Markov chain is "a stochastic mode describing a sequence of possible events in which the probability of each event depends only on the state attained in the previous event". In agricultural research, it's relatively rare to forecast the development of agricultural industrial structure. Obviously, for each state, the agricultural industrial structure depends only on the state attained in the previous agricultural industrial structure. Therefore, Markov model is suitable for the study of the development of agricultural industrial structure.

\section{B. Markov Model Basics}

Suppose that there are $n$ states $S_{1}, S_{2}, \ldots, S_{n}$ in the system. The process starts in one of these states and moves successively from one state to another. The probability that system from state $S_{i}$ to state $S_{j}$ in one step is denoted by $p_{i j}(i, j=1,2, \ldots, n)$. Noted the one-step transition probability matrix as $P=\left(p_{i j}\right)$,

$$
\sum_{j=1}^{n} p_{i j}=1, \text { and } 0 \leq p_{i j} \leq 1
$$

The transition probability is $P(k)$ after $k$ step, $P(k)=P(k-1) \cdot P=P^{k}$. Assume that the system is in $\mathrm{S}(0)$ at the beginning, $\mathrm{S}(0)=\left[S_{1}(0), S_{2}(0), \ldots, S_{n}(0)\right]$, where $S_{j}(0)(j=1,2, \ldots, n)$ is the probability of in state $S_{j}$, then $\sum_{j} S_{j}(0)=1$. The system is $\mathrm{S}(k)$ after $\mathrm{k}$ step. So we have $\mathrm{S}(k)=\mathrm{S}(k-1) \cdot P=\mathrm{S}(0) \cdot P^{k}$.

\section{Application of Markov in Agricultural Industrial Structure}

Assume that planting, animal husbandry, fishery and other industries is the four states of agricultural industries. Note the error of the ratio of the $j^{\text {th }}$ industry output value to the total agricultural output value in $t$ is

$$
e_{j}(t)=S_{j}(t)-\sum_{i=1}^{4} S_{i}(t-1) \hat{p}_{i j}, \quad j=1,2,3,4
$$

$$
Q=\sum_{j=1}^{4} Q_{j}=\sum_{j=1}^{4} \sum_{t}\left[e_{j}(t)\right]^{2}
$$

We minimize $Q$ in this model, and have the nonlinear programming:

$$
\begin{aligned}
\min Q= & \sum_{j=1}^{4} \sum_{t}\left[S_{j}(t)-\sum_{i=1}^{4} S_{i}(t-1) \hat{p}_{i j}\right]^{2} \\
& \left\{\begin{array}{l}
\sum_{i=1}^{4} \hat{p}_{i j}=1 \\
\hat{p}_{i j} \geq 0 \quad i, j=1,2,3,4
\end{array}\right.
\end{aligned}
$$

We can obtain $P$ by least squares,

$$
\begin{gathered}
\text { Where } Y=X_{1}{ }^{T} X_{1}, M=X_{1}{ }^{T} X_{2} \\
X_{1}=\left[\begin{array}{ccc}
S_{0}(1) & \cdots & S_{0}(t) \\
\vdots & & \vdots \\
S_{3}(1) & \cdots & S_{3}(t)
\end{array}\right], X_{2}=\left[\begin{array}{ccc}
S_{2}(1) & \cdots & S_{2}(t) \\
\vdots & & \vdots \\
S_{4}(1) & \cdots & S_{4}(t)
\end{array}\right]
\end{gathered}
$$

D. Analysis of Agricultural Industrial Structure Development

According to the data published by the Statistical Yearbook, we obtain

TABLE VI. 2007-2015 PROPORTION IN VARIOUS AGRICULTURAL INDUSTRIES IN SHUNDE

\begin{tabular}{ccccc}
\hline \hline Year & Planting & $\begin{array}{c}\text { Animal } \\
\text { husbandry }\end{array}$ & Fishery & Other \\
\hline 2007 & 0.234327 & 0.143625 & 0.619117 & 0.002931 \\
2008 & 0.196895 & 0.149884 & 0.610708 & 0.042513 \\
2009 & 0.212729 & 0.142794 & 0.601609 & 0.042868 \\
2010 & 0.220108 & 0.146549 & 0.590315 & 0.043028 \\
2011 & 0.217452 & 0.167114 & 0.570589 & 0.044845 \\
2012 & 0.223827 & 0.150421 & 0.581227 & 0.044525 \\
2013 & 0.236575 & 0.110625 & 0.606246 & 0.046555 \\
2014 & 0.214513 & 0.081298 & 0.651209 & 0.052979 \\
2015 & 0.223436 & 0.054987 & 0.655661 & 0.065915 \\
\hline
\end{tabular}

In the table, $t=9$, and

$$
\mathrm{S}(0)=\left[\begin{array}{llll}
0.234327, & 0.143625, & 0.619117, & 0.002931
\end{array}\right]
$$

With formula (11), we obtain the estimated transition probability matrix

$$
\hat{P}=\left[\begin{array}{cccc}
0.2283 & 0 & 0.7006 & 0.0711 \\
0.1760 & 0.8240 & 0 & 0 \\
0.1273 & 0.1333 & 0.6704 & 0.0690 \\
0.5176 & 0 & 0.3581 & 0.1244
\end{array}\right]
$$

Thus we predict the development of the agricultural industry structure in Shunde by using Markov model,

TABLE VII. APPLICATION TO THE DEVELOPMENT OF AGRICULTURAL INDUSTRIAL STRUCTURE WITH MARKOV

\begin{tabular}{c|c|c|c|c|c|c|c|c}
\hline \hline \multirow{2}{*}{ Year } & \multicolumn{2}{|c|}{ Planting } & \multicolumn{2}{c|}{ Animal husbandry } & \multicolumn{3}{c|}{ Fishery } & \multicolumn{2}{c}{ Other } \\
\cline { 2 - 8 } & prediction & relative error & prediction & relative error & prediction & relative error & prediction & relative error \\
\hline 2007 & 0.234327 & 0.000000 & 0.143625 & 0.000000 & 0.619117 & 0.000000 & 0.002931 & 0.000000 \\
\hline 2008 & 0.196035 & 0.000522 & 0.152022 & 0.000522 & 0.610137 & 0.000522 & 0.041806 & 0.000522 \\
\hline 2009 & 0.217134 & 0.014727 & 0.151263 & 0.014727 & 0.587437 & 0.014727 & 0.044166 & 0.014727 \\
\hline 2010 & 0.219642 & -0.009012 & 0.134124 & -0.009012 & 0.599736 & -0.009012 & 0.046498 & -0.009012 \\
\hline 2011 & 0.221752 & -0.028276 & 0.133827 & -0.028276 & 0.598582 & -0.028276 & 0.045839 & -0.028276 \\
\hline 2012 & 0.225244 & 0.002778 & 0.156415 & 0.002778 & 0.577436 & 0.002778 & 0.040905 & 0.002778 \\
\hline 2013 & 0.224076 & 0.015069 & 0.134787 & 0.015069 & 0.595933 & 0.015069 & 0.045203 & 0.015069 \\
\hline
\end{tabular}




\begin{tabular}{c|c|c|c|c|c|c|c|c}
\hline 2014 & 0.222763 & 0.012469 & 0.082578 & 0.012469 & 0.638927 & 0.012469 & 0.055733 & 0.012469 \\
\hline 2015 & 0.218890 & -0.002945 & 0.058657 & -0.002945 & 0.659376 & -0.002945 & 0.063076 & -0.002945 \\
\hline
\end{tabular}
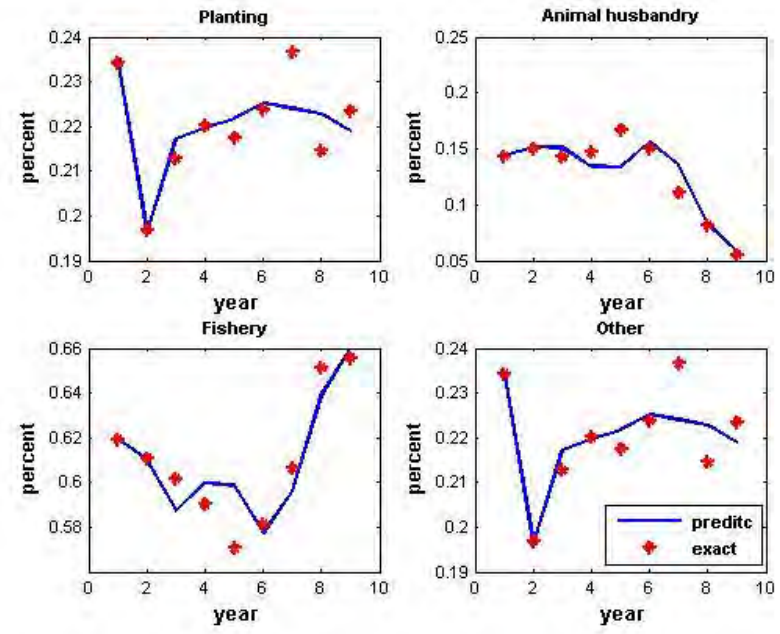

Fig. 1. Application with Markov

From the result, the prediction of the development of agricultural industrial structure by Markov model fits very close to the actual situation, and some errors can even be ignored.

\section{E. Prediction of Agricultural Industrial Structure in Shunde}

By the above results, we predict the development of agricultural industrial structure in Shunde from 2016 to 2020 as follows:

TABLE VIII. PREDICTION BY MARKOV FORECASTING MODEL

\begin{tabular}{ccccc}
\hline \hline Year & Planting & $\begin{array}{c}\text { Animal } \\
\text { husbandry }\end{array}$ & Fishery & Other \\
\hline 2016 & 0.1769 & 0.1362 & 0.6180 & 0.0689 \\
\hline 2017 & 0.1787 & 0.1946 & 0.5629 & 0.0638 \\
\hline 2018 & 0.1797 & 0.2354 & 0.5254 & 0.0595 \\
\hline 2019 & 0.1801 & 0.2640 & 0.4994 & 0.0564 \\
\hline 2020 & 0.1769 & 0.1362 & 0.6180 & 0.0689 \\
\hline
\end{tabular}

The result shows the proportion of fishery will increase while planting and other will decline with a weak trend.

\section{CONCLUSIONS}

\section{A. Analysis of Model Results}

Based on the forecasting results above for the development of agriculture, the agricultural development in Shunde is progressing rapidly, especially during the period from 2010 to 2013. However, from the study of the development of agricultural structure, we can find that although the agricultural economic development of Shunde still continue to expand, the development speed is gradually slowing down, the structure is tending to be single, and the number of the agricultural practitioners is decreasing sharply. Planting and other industries decline with a weak trend and the development of fishery still keep progressing in trend. Problems have become increasingly prominent with development while agricultural economy in Shunde is developing. The bottleneck of agricultural economic development in Shunde is increasing day by day, and the development trend will be curbed.

\section{B. Analysis of Research Results}

In order to find out the bottleneck of the agricultural economic development in Shunde, and to provide practical recommendations, we conducted surveys and interviews with local farmers and local non-agricultural personnel in Beijiao Town, Shunde. Excluding invalid records, in this paper, we adopted a total of 20 detailed interview samples, and summarized and concluded the main characteristics of the current Beijiao planting as follows:

(1) The proportion of leased land used for planting reached $100 \%$, and the government's land acquisition policy and high rents have led to a significant contraction in planting.

(2) Agricultural practitioners in the future generation is very small, and there is a tendency of gradually aging and reducing for the peasants.

(3) The local market turning gradually saturated and its competitiveness turning increased, while the sales of local agricultural practitioners are geting mostly exported to wholesalers so that the interests of farmers were weakened. In addition, the flower market in the local planting industry occupies a large part, while flowers, as an ornamental product, aren't necessities, resulting in a large change in market demand.

(4) The local government is more inclined to industrial development, less investment in agricultural development, and less favorable policies for local agriculture.

(5) It is difficult to popularize automatic planting in the local area. However, ordinary planting takes a lot of time and effort, and the labor costs also gradually increase, which leads to the limited local planting development.

\section{Suggestions}

In response to the bottleneck in the agricultural development, we propose the following suggestions:

(1) It is recommended that the local government implement some policies of encouraging youth to engage in scientific agriculture. On the one hand, combining with the convenience brought by scientific development will be more conducive to the development of agriculture. For another, it is also beneficial to ease the work problem.

(2) It is suggested that the local government rationally develop industrial land, remain planned land for agricultural purposes, and reasonably collect rental fees. At the same time, it is recommended that local governments actively launch a subsidy policy for senior citizens to provide life insurance for senior farmers.

(3) It is recommended that the local government encourage the modernization of the local agricultural industry and make full use of the convenient technologies such as the 
Internet to promote local specialty industries and develop local agricultural industries.

(4) It is recommended that local governments take advantage of the local characteristics and technology of mulberry fish ponds to further optimize and improve the fishery industry structure, so as to further develop the local fishery industry and promote local agricultural development.

\section{ACKNOWLEDGMENT}

This work was supported by the Fundamental Research Funds for the Central Universities (Grant No. 21617417).

\section{REFERENCES}

[1] J.M.Bates, C.W.J.Granger. The Combination of Forecasts[J]. Journal of Operational Research Society. 1969,20(4):451-468.

[2] Deng J.L.. Introduction to Grey System Theory[J]. The Journal of Grey System(UK). 1989,1(1):1-24.

[3] Ju Jinyan, Zhu Rongxin. Prediction Structure of Agriculture Output Value Based on Markov Model[J]. Mathematics in Practice and Theory. 2013,43(22):65-70.
[4] Zeng Bo, Liu Sifeng, Fang Zhigeng, etc. Grey Combined Forecast Models and Its Application[J]. Chinese Journal of Management Science. 2009,17(5):150-155.

[5] Shi Ping, Tang Jun. Application of $\operatorname{GM}(1, n)$ Prediction Model in Economic Forecasting: Taking Economic Development of Baotou as an Example[J]. Journal of Inner Mongolia University (Natural Science Edition). 2015,46(1):43-47.

[6] Zhang Hao. Study on Forecasting the Structure of Agricultural Production Value in China[D]. Northeast,China: Northeast Agricultural University,2011.

[7] Wang Wei, Li Yu. The Application of Optimal Combination Forecast in the Talent Requirement Forecast of Sichuan[J]. Value Engineering. 2005,(3):9-12.

[8] Zhu Chunjiang, SINGH Surendre P, COMER Sammy L. Studies on Optimization Combination C Forecasting for Agricultural Gross Domestic Product of China[J]. Hubei Agricultural Sciences. 2013,52(23):5930-5933.

[9] Tang Xiaowo, Zeng Yong, Cao Changxiu. The Estimation of Transition Probability of Markov Chains in Market Forecasting[J]. Journal of UEST of China. 1994, 23(6):643-648. 